\title{
Chinese protein scientists met in Hefei for the 4th Chinese Protein Society Symposium
}

\author{
LI GenXi ${ }^{1 *} \&$ CHANG Zeng $\mathrm{Yi}^{2^{*}}$ \\ ${ }^{1}$ Department of Biochemistry and State Key Laboratory of Pharmaceutical Biotechnology, Nanjing University, Nanjing 210093, China; \\ ${ }^{2}$ School of Life Sciences and Center of Protein Science, Peking University, Beijing 100871, China
}

Received October 30, 2013; accepted October 31, 2013; published online November 6, 2013

Citation: $\quad$ Li G X, Chang Z Y. Chinese protein scientists met in Hefei for the 4th Chinese Protein Society Symposium. Sci China Life Sci, 2013, 56: 1154-1155, doi: 10.1007/s11427-013-4571-4

Some 600 scholars and students in protein science from around 40 institutions across China gathered at the 4th Symposium of the Chinese Protein Society held during October 12-14, 2013, in the city of Hefei, Anhui Province, China, with the theme "Protein and Human Health". This symposium was organized by the Chinese Protein Society, and locally sponsored by the University of Science and Technology of China and Anhui University. The topics of this symposium covered almost all areas in protein science, from structure to function, from mechanism to regulation, from single molecule to computer simulation, from in vitro (in the test tube) to in vivo (in living cells), from qualitative to quantitative studies, from basic research to medical application, and from protein engineering to agriculture.

Prof. Li GenXi, Secretary-General of the Chinese Protein Society and this symposium, presided the opening and closing ceremonies. Those who addressed the opening ceremony included Academician Wang ZhiXin (President of the Chinese Society for Biochemistry and Molecular Biology), Prof. Zhu ChangFei (vice-President of the University of Science and Technology of China), Academician Wang ZhiZhen (Chih-chen) (the former President of the Chinese Protein Society) and Prof. Chang ZengYi (the current President of the Society). Academician Wang ZhiZhen gave a historical perspective on the development of the Chinese Protein Society since its foundation in 2005. As emphasized by Academician Wang, the mission of the Chinese Protein

*Corresponding author (email: genxili@nju.edu.cn; changzy@pku.edu.cn)
Society is to facilitate the scholarly exchange and cooperation between protein scientists inside and outside China, from all disciplines and backgrounds. She wanted Chinese scientists as a whole to ultimately "lead" the world in certain areas. She emphasized that Chinese scientists should stick to the true course of scientific research, devoting their "loyalty, assiduousness and whole-heartedness" to research and education. She also wanted Chinese scientists to strictly abide by the professional ethnics in conducting scientific research. Academician Wang ZhiZhen also appealed to all the established scholars to have great patience, enthusiasm and responsibility in educating young scholars and to actively spread science to the general public. Meanwhile, she also hoped that as a scientist one should gain a taste for art.

This symposium was embraced with great passion by researchers from very different backgrounds. During the two-and-a-half day meeting, the plenary talks were highly regarded and were given by distinguished scholars, including Prof. Wang XiaoDong, Director of the National Institute of Biological Sciences and a member of the US National Academy of Sciences; Prof. Niu LiWen, the former Dean of School of Life Sciences at the University of Science and Technology of China, and currently a vice-President of Anhui University; Prof. Shao Feng, winner of the 2013 Young Investigator Award of the Protein Society; Prof. Shi YiGong, Dean of School of Life Sciences at Tsinghua University and a foreign associate of the US National Academy of Sciences; Prof. Shi YunYu, member of the Chinese Academy of Sciences; Prof. Rao Yi, the former Dean of 
School of Life Sciences, Peking University; and Prof. Sui SenFang, member of the Chinese Academy of Sciences. In addition, another 33 invited speakers from 23 institutions covered a wide range of areas of protein research.

The organizing committee also selected 15 speakers from those young scholars who submitted an abstract. To provide a chance for the students to talk in front of the audiences and as a tradition of this symposium series, the committee organized three student sessions, with 24 graduate students selected who talked about their research work.

At the closing ceremony, as the local host of this symposium, Prof. Niu LiWen, vice-President of Anhui University, thanked all the attendees for their participation; Prof. Chang ZengYi, Chair of the organizing committee of this symposium, expressed his appreciation to the great efforts made by Prof. Teng MaiKun, vice-Dean, and Prof. Zang JianYe, both of School of Life Sciences at the University of Science and Technology of China, for their time and effort in arranging the details for the meeting. He wanted Chinese protein scientists to mark their calendar for the 4th Symposium of the Asia Pacific Protein Association (APPA) to be held during May 14-17, 2014, in Jeju Island, Korea (Prof. Chang ZengYi is currently serving as the President of APPA). He also informed the attendees that the Chinese Protein Society is bidding to host the Protein Society (an international organization) symposium of 2017 in Shanghai, China. At the closing ceremony, the best student oral and poster presentations were announced and awarded. In the end, Academician Wang ZhiZhen, honorable chair of the organizing committee of this symposium, summarized the highlights of this symposium, as characterized by its interdisciplinary nature. As a tradition of the Chinese Protein Society symposium, in the afternoon of October 14, Academician Wang ZhiZhen, together with several renowned scholars including Profs. Shi YiGong, Chang ZengYi and Dong YuHui went to Hefei No. 1 High School, the alma mater of Nobel Laureate Yang Chen Ning, and gave a seminar to about 1000 high school students, covering different aspects of protein and protein research. It was greatly welcomed by the students.

Open Access This article is distributed under the terms of the Creative Commons Attribution License which permits any use, distribution, and reproduction in any medium, provided the original author(s) and source are credited. 